\title{
TRANSIENT MICRO-NANO HEAT CONDUCTION ANALYSIS USING A COMBINATION OF THE FINITE DIFFERENCE METHOD, THE COLLOCATION MESHFREE METHOD AND THE DISCRETE ORDINATE METHOD
}

\author{
SAEID ZAHIRI ${ }^{1}$, JIEMING SONG $^{1}$, HUA BAO $^{1} \&$ YONGXING SHEN $^{1,2}$ \\ ${ }^{1}$ University of Michigan-Shanghai Jiao Tong University Joint Institute, Shanghai Jiao Tong University, China \\ ${ }^{2}$ State Key Laboratory of Metal Matrix Composites, Shanghai Jiao Tong University, China
}

\begin{abstract}
For studying transient heat conduction problems in the micro-nano scale, the standard heat diffusion equation is no longer applicable, and the time-dependent Boltzmann transport equation (BTE) needs to be solved. Although mesh-based numerical methods such as the finite element method (FEM) and the finite volume method (FVM) are often employed to solve the BTE, the collocation meshfree method has special advantages since it does not require numerical integration. In this work, the collocation meshfree method and the discrete ordinate method (DOM) are implemented to discretize the spatial and angular domains, respectively, while the explicit finite difference method (FDM) is used for advancing in time. Such a method is used to solve the transient BTE for a square domain with prescribed temperature and adiabatic boundary conditions. Our results are consistent with transient heat transfer problems solved by FVM approach.

Keywords: transient Boltzmann transport equation, collocation meshfree method, discrete ordinate method, finite difference method, ballistic and diffusive regimes.
\end{abstract}

\section{INTRODUCTION}

In transient heat transfer problem, the heat diffusion equation is no longer valid for small scale geometry and ballistic heat transfer regime, although for the diffusive heat transfer regime the results are acceptable. To solve this problem, the phonon BTE is still the state-of-the-art method [1]-[4]. For transient problems, there are some numerical methods to solve the BTE; e.g., FEM [5], [6], FDM [7], and FVM [8], [9]. Some of the previous works are reviewed as follows.

The transient phonon transport is considered in two-dimensional silicon film problems and was solved by Yilbas and Mansoor [10]. They used the FDM and an implicit scheme to solve the frequency-dependent BTE for a square domain. Cobos et al. analyzed geometry irradiated by photon beams by solving the time-dependent BTE with the time step given by a modified Courant-Friedrichs-Lewy condition [3]. Guo and Xu discretized the transient BTE with a second-order finite volume formulation and used the discrete unified gas kinetic scheme [11]. Joshi and Majumdar modeled the ballistic to diffusive phonon transport regimes by solving the equation of phonon radiative transfer for a 1-D geometry. This equation was solved by using the explicit upstream differencing method [12]. In 2015, Hamian et al. [5] used the commercial software COMSOL to study a square domain and solved the BTE by using FEM and DOM.

In our previous work [4], we applied the collocation meshfree method and the DOM to solve the steady state gray BTE. This work is built upon the work in Zahiri et al. [4], in which we used a combination of the FDM, the collocation meshfree method and the DOM to analyze transient heat conduction problems in the sub-continuum scale for a wide range of Knudsen numbers $(K n)$. These simulations are for 1-D and 2-D geometries and are implemented by discretizing the time dependent gray BTE. 


\section{BOUNDARY CONDITIONS AND GOVERNING EQUATION}

The time-dependent form of the gray BTE for phonon transport without any energy source is [5]

$$
\frac{\partial e}{\partial t}+\nabla \cdot(\mathbf{v} e)=\frac{\partial e}{\partial t}+\nabla \cdot\left(\mathbf{s} v_{g} e\right)=\left(\frac{\partial e}{\partial t}\right)_{\text {collision }}
$$

Here $e(\mathbf{x}, \mathbf{s}, t)$ is the phonon energy density and is a function of position $\mathbf{x}$, direction of group velocity $\mathbf{s}$ and time $t$. The group velocity vector is denoted by $\mathbf{v}, v_{g}=|\mathbf{v}|$ and $\nabla \cdot$ is the divergence operator [4].

By using the Bhatnagar-Gross-Krook model, the right side of eqn (1) is simplified as $\left(\frac{\partial e}{\partial t}\right)_{\text {collision }}=\frac{e^{0}-e}{\tau}$, in which $e^{0}$ is the phonon energy density in equilibrium and is calculated by: $e^{0}(\mathbf{x}, t)=\frac{1}{4 \pi} \int_{\Omega} e(\mathbf{x}, \mathbf{s}, t) d \Omega$ over all solid angles $\Omega$.

In addition, two different boundary conditions are prescribed in this work: the first is the prescribed temperature boundary $\left(T_{b}\right)$ condition: $e(\mathbf{x}, \mathbf{s})=\frac{C}{4 \pi}\left(T_{b}-T_{r e f}\right)$ for incoming directions; i.e., $\mathbf{s}$ such that $\mathbf{s} \cdot \mathbf{n}<0$, where $\mathbf{n}$ is the unit normal vector of the boundary and $C$ is the specific heat [4]. The second is the specular (adiabatic) boundary condition in which each incident phonon is reflected such that $e(\mathbf{x}, \mathbf{s})=e\left(\mathbf{x}, \mathbf{s}_{r}\right)$ for incoming directions such that $\mathbf{s} \cdot \mathbf{n}<0$, where $\mathbf{s}_{\mathbf{r}}=\mathbf{s}-(2 \mathbf{s} \cdot \mathbf{n}) \mathbf{n}[4],[12]$.

\section{NUMERICAL METHOD}

The finite difference and collocation meshfree methods are described here to solve the gray BTE for temporal and spatial discretizations, respectively. The collocation meshfree method does not need a mesh and instead, field nodes scattered in the domain are used to interpolate the solution. Also for angular discretization we use the discrete ordinate method (DOM) [13].

To solve the transient problem, it is possible to use the FDM to discretize the term $\frac{\partial e}{\partial t}$ of eqn (1) as [14]:

$$
\frac{\partial e}{\partial t}=\frac{e^{n+1}-e^{n}}{\Delta t}
$$

where $e^{n+1}$ is the data for the next time step $n+1$ when $n$ is in the present time step and $\Delta t$ is the time step size. For the explicit Euler method, all other variables in the equation should be in the time step $n$. Therefore, using eqns (1) and (2) yields

$$
e^{n+1}=e^{n}+\Delta t\left(-\nabla \cdot\left(\mathbf{s} v_{g} e^{n}\right)+\frac{\left(e^{0}\right)^{n}-e^{n}}{\tau}\right)
$$


To discretize the angular domain, the DOM is used and each hemisphere is divided by $N=4 \times N_{\theta} \times N_{\varphi}$ solid angles where $\theta$ is the polar angle and $\varphi$ is the azimuthal angle [4], [13].

To perform spatial discretization by a meshfree method, interpolation methods are used. The radial point interpolation method (RPIM) is selected to make shape functions [14], [15]. For a specific direction $\mathbf{s}_{j}$, the energy density field to be solved is given by $e\left(\mathbf{x}, \mathbf{s}_{j}\right)=\sum_{i=1}^{n} \phi_{i} e_{i}\left(\mathbf{s}_{j}\right)$ where $e_{i}\left(\mathbf{s}_{j}\right)=e\left(\mathbf{x}_{i}, \mathbf{s}_{j}\right)$ and $\phi_{i}$ is the shape function for the $i$-th node in the support domain [4]. The derivatives of $e(\mathbf{x})$ are computed as

$$
\frac{\partial e}{\partial x}\left(\mathbf{x}, \mathbf{s}_{j}\right)=\sum_{i=1}^{n} \frac{\partial \phi_{i}}{\partial x} e_{i}\left(\mathbf{s}_{j}\right)
$$

and $\partial e / \partial y$ can be obtained analogously. The accuracy of interpolation depends on the size of the support domain and how many nodes are selected therein [16]. By using eqns (3) and (4), the final formula of BTE for 2-D time-dependent collocation meshfree method is obtained [4]:

$$
\begin{aligned}
& e_{k}^{n+1}\left(\mathbf{s}_{j}\right)= \\
& e_{k j}^{n}\left(\mathbf{s}_{j}\right)+\Delta t\left(-\left(s_{j x} v_{g} \sum_{k=1}^{n} \frac{\partial \phi_{k}}{\partial x}\left(x_{i}\right) e_{k}^{n}\left(\mathbf{s}_{j}\right)+s_{j y} v_{g} \sum_{k=1}^{n} \frac{\partial \phi_{k}}{\partial y}\left(x_{i}\right) e_{k}^{n}\left(\mathbf{s}_{j}\right)\right)+\frac{\left(e_{i}^{0}\right)^{n}-e_{i}\left(\mathbf{s}_{j}\right)^{n}}{\tau}\right),
\end{aligned}
$$

where $\mathbf{s}_{j}=\left(s_{j x}, s_{j y}\right)$ and $e_{i}^{0}=e^{0}\left(\mathbf{x}_{i}\right)$. To satisfy the boundary conditions, it is just required to put calculated $e\left(\mathbf{x}_{b}, \mathbf{s}_{j}\right)$ from the previous section equations for the phonon energy density for corresponding node and direction in the boundary [4].

\section{RESULTS}

In this section, we solve a few transient sample problems in a square domain $[0, L] \times[0, L]$ with the proposed method and discuss the results. We will study two sets of boundary conditions:

a) For the first set of examples, we prescribe on the left boundary $(x=0)$ the condition $T=T_{2}$, for the right boundary $(x=L)$ the condition $T=T_{1}$, and top and bottom boundaries $(y=0$ and $y=L)$ the specular adiabatic boundary condition. The solution is expected to only depend on $x$ and $t$.

b) In the second set of examples, the left and right boundaries are the same as in the first set, while for the top and bottom boundaries we assume $T=T_{1}$; i.e., the same temperature as the right boundary.

Besides the proposed method (denoted Meshfree in the figures), we will also compare a method where the spatial discretization is with a cell-centered finite volume method instead, denoted as FVM.

We will compare the results with different Knudsen numbers $K n=l / L$, where $l$ is mean free path and $L$ is the domain side length. Also the non-dimensional length $\left(x^{*}=x / L\right.$, 
$\left.y^{*}=y / L\right)$ and the non-dimensional temperature $\left(T^{*}=\left(T-T_{1}\right) /\left(T_{2}-T_{1}\right)\right)$ are calculated for different dimensionless time $\left(t^{*}=t / \tau\right)$ for verification. According to a grid study given by Zahiri et al. [4], the results for $21 \times 21$ and $41 \times 41$ nodes have reasonable agreement with FEM results [5] and, in the diffusive regime, with the analytic solution of Fourier heat conduction.

We generally plot the non-dimensional temperature distribution at the midline $\left(y^{*}=0.5\right)$. In case a), we will study $K n$ values between 0.01 and 100 , representing the diffusive to ballistic phonon transport regimes. The number of solid angle divisions $(N)$ is 256 for the upper hemisphere, and the lower hemisphere is assumed to be symmetric to the upper hemisphere.

The results for case a) are shown in Fig. 1 and Fig. 2, with $K n=0.01$ and 100, respectively. In Fig. 1, the results from the analytical solution of Fourier heat transfer are also plotted for verification purpose. It is clear that the meshfree method agrees better with the Fourier heat conduction solution than FVM with the same number of nodes and discretized solid angles.

Although our transient results for different time steps do not match well with those of the FVM, the long-time solution, meaning when the results no longer change with time. Both the FVM and the meshfree method are well consistent with the analytical solution of the heat diffusion equation.

In Fig. 2, the distribution of non-dimensional temperature for $K n=100$ and for different time steps are presented. The FEM and meshfree method results for $K n=100$ for different time steps are in better agreement than $K n=0.01$. Both the steady state temperature solution and the long-time results for meshfree and FVM demonstrate temperature jumps at the right and left boundaries. This phenomenon is known as ballistic phonon transport regime.

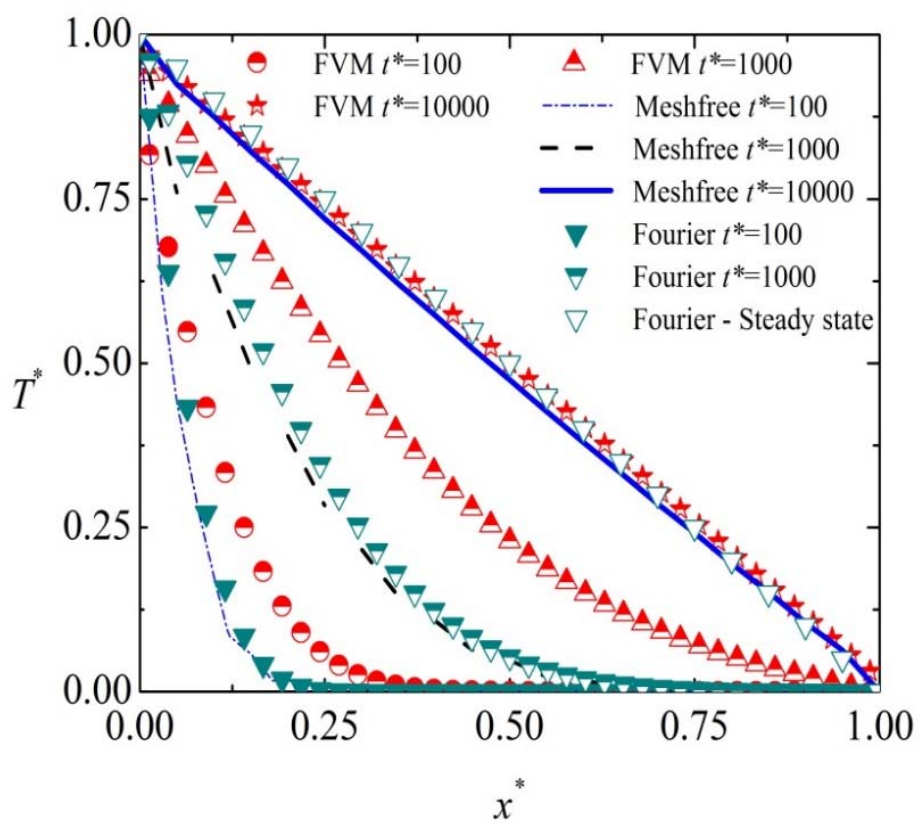

Figure 1: Temperature distribution along the midline for specular adiabatic boundary conditions for $K n=0.01$. 


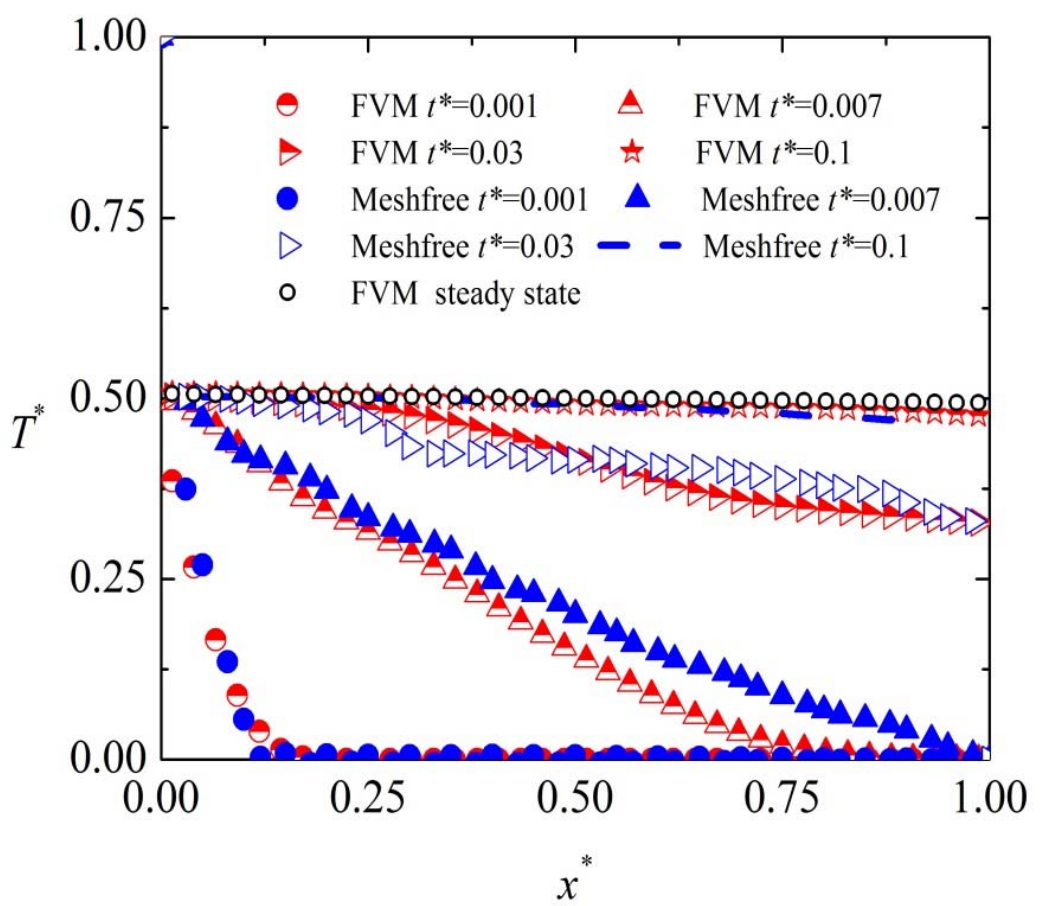

Figure 2: Temperature distribution along the midline for specular adiabatic boundary conditions for $K n=100$.

For case b) we consider prescribed temperature boundary conditions in all boundaries of the square domain and study the transient heat conduction in the following. The left boundary is at a higher temperature, while the other sides have a lower temperature. The results are illustrated in Figs 3 and 4. We compared the results with our results obtained from the FVM. Also, we solved the problem for different time steps, and yet they do not have exactly the same curves.

In the results for steady state and a long-time, overlapping is observed in meshfree results. However, there are oscillations in steady state when these oscillations can be seen also in the meshfree results for a long-time because of ray effects but not in FVM. It means that the ray effects due to the finite angular discretization for the problem solved by using meshfree method with the same number of nodes and same number of discretized angles is more visible than in FVM.

In Fig. 4 results for $K n=1$ are presented. The oscillations are smaller than $K n=10$ and this figure and Fig. 3 show that the ray effects for higher $K n$ has a greater effect in comparison to smaller $K n$. However, the results for the long-time have less temperature jump than the results from higher $K n$ in the boundaries. 


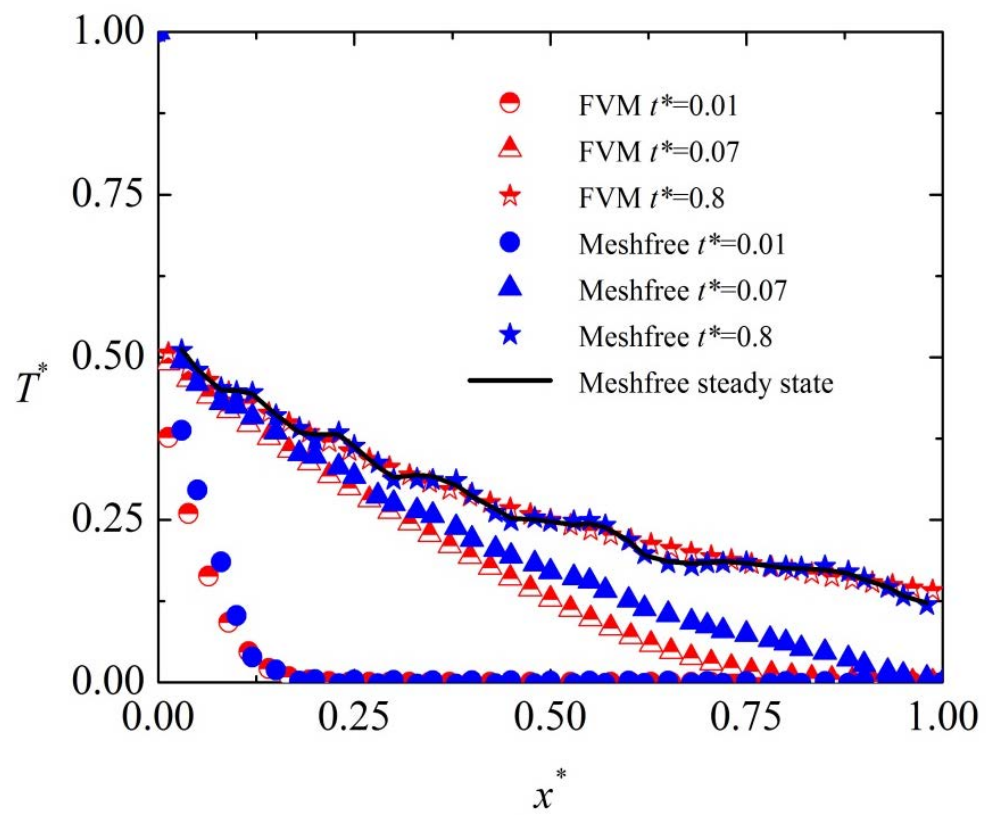

Figure 3: Results for $K n=10$ for constant temperature boundary conditions.

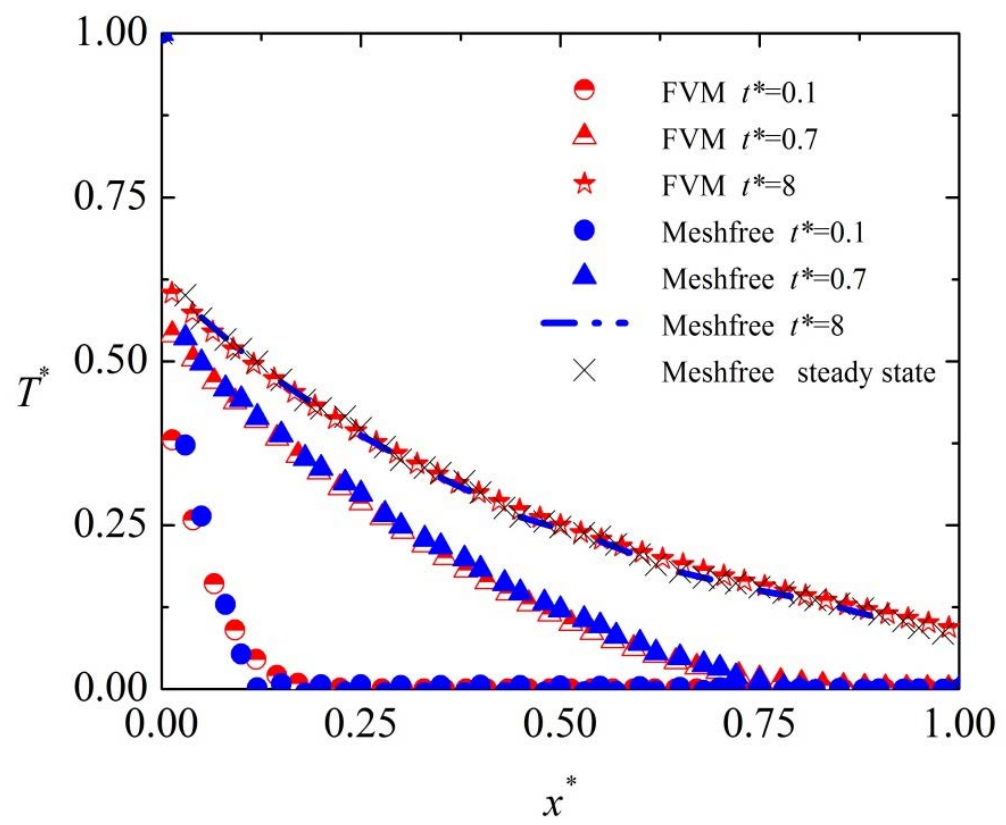

Figure 4: Results for $K n=1$ for constant temperature boundary conditions. 


\section{CONCLUSION}

In this work, transient heat transfer problems in sub-continuum geometries have been studied by a numerical method combining the FDM, collocation meshfree method and the DOM due to the necessity of time, spatial, and angular discretizations to solve the time-dependent phonon gray BTE. The results for higher $K n$ numbers demonstrate reasonable agreement with our FVM results for time steps prior to convergence results for a long-time. Also, different transient heat transfer regimes from ballistic to diffusion have been studied in this work. We found that the results for meshfree and FVM at the same number of field nodes and discretized solid angles for high $K n$ numbers agree well, although for low $K n$ numbers, meshfree has better agreement than the FVM with analytical solution of Fourier heat transfer equation results in the diffusive regime.

\section{ACKNOWLEDGEMENTS}

This work is supported by the National Natural Science Foundation of China (grant numbers 51306111 and 11402146) and Shanghai Municipal Natural Science Foundation (grant number 13ZR1456000). YS acknowledges the financial support from the Young 1000 Talent Program.

\section{REFERENCES}

[1] Murthy, J.Y. \& Mathur, S.R., Computational heat transfer in complex systems: A review of needs and opportunities. Journal of Heat Transfer, 134(3), pp. 1-12, 2012.

[2] Chattopadhyay, A. \& Pattamatta, A., A comparative study of submicron phonon transport using the Boltzmann transport equation and the lattice Boltzmann method. Numerical Heat Transfer, Part B: Fundamentals, 66(4), pp. 360-379, 2014.

[3] Cobos, A.C., Poma, A.L., Alvarez, G.D. \& Sanz, D.E., Vectorization of the timedependent Boltzmann transport equation: Application to deep penetration problems. Radiation Physics and Chemistry, 127, pp. 102-114, 2016.

[4] Zahiri, S., Shao, C., Shen, Y. \& Bao, H., Collocation meshfree method to solve the gray phonon Boltzmann transport equation. Numerical Heat Transfer, Part B: Fundamentals, 2016. DOI: 10.1080/10407790.2016.1215719.

[5] Hamian, S., Yamada, T., Faghri, M. \& Park, K., Finite element analysis of transient ballistic-diffusive phonon heat transport in two-dimensional domains. International Journal of Heat and Mass Transfer, 80, pp. 781-788, 2015.

[6] Lee, P., Yang, R. \& Maute, K., An extended finite element method for the analysis of submicron heat transfer phenomena. Multiscale Methods in Computational Mechanics, Springer Netherlands, pp. 195-212, 2011.

[7] Heino, P., Lattice-Boltzmann finite-difference model with optical phonons for nanoscale thermal conduction. Computers \& Mathematics with Applications, 59(7), pp. 2351-2359, 2010.

[8] Kim, M.Y., Baek, S.W. \& Park, J.H., Unstructured finite-volume method for radiative heat transfer in a complex two-dimensional geometry with obstacles. Numerical Heat Transfer, Part B: Fundamentals, 39(6), pp. 617-635, 2001.

[9] Murthy, J.Y. \& Mathur, S.R., Computation of sub-micron thermal transport using an unstructured finite volume method. Journal of Heat Transfer, 124(6), pp. 1176-1181, 2002.

[10] Yilbas, B.S. \& Mansoor, S.B., Transient effects of phonon transport in twodimensional silicon film. Numerical Heat Transfer, Part A: Applications, 62(9), pp. 742-760, 2012. DOI: 10.1080/10407782. 
[11] Guo, Z. \& Xu, K., Discrete unified gas kinetic scheme for multiscale heat transfer based on the phonon Boltzmann transport equation. International Journal of Heat and Mass Transfer, 102, pp. 944-958, 2016.

[12] Joshi, A.A. \& Majumdar, A., Transient ballistic and diffusive phonon heat transport in thin films. Journal of Applied Physics, 74, pp. 31-39, 1993.

[13] Donmezer, F.N., Lattice Boltzmann and discrete ordinates methods for phonon transport modeling: A comparative study. ASME 2011 International Mechanical Engineering Congress and Exposition, D. Singh, W. James, A. Christensen, S. Graham \& J.Y. Murthy, American Society of Mechanical Engineers, pp. 333-343, 2011.

[14] Zahiri, S., Using meshfree weak-strong form method for a 2-D heat transfer problem. ASME 2009 International Mechanical Engineering Congress and Exposition, F. Daneshmand \& M.H. Akbari, IMECE2009-12525, pp. 643-651, 2009.

[15] Zahiri, S., Meshfree Methods. Numerical Analysis-Theory and Application, INTECH Open Access Publisher, 2011.

[16] Liu, G.R. \& Gu, Y.T., An Introduction to Meshfree Methods and Their Programming, Springer Science \& Business Media, 2005. 